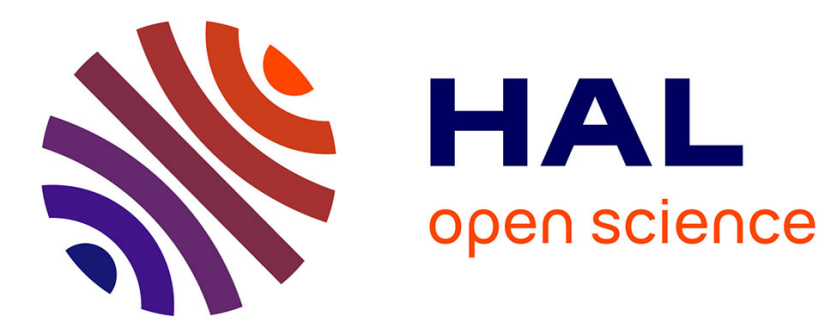

\title{
An Emergent Model for Mimicking Human Neuronal Pathways in Silico
}

\author{
Önder Gürcan
}

\section{To cite this version:}

Önder Gürcan. An Emergent Model for Mimicking Human Neuronal Pathways in Silico. 12th European Conference on Artificial Life (ECAL 2013), Sep 2013, Taormina, Italy. pp. 1172-1173. hal01193097

\section{HAL Id: hal-01193097 \\ https://hal.science/hal-01193097}

Submitted on 4 Sep 2015

HAL is a multi-disciplinary open access archive for the deposit and dissemination of scientific research documents, whether they are published or not. The documents may come from teaching and research institutions in France or abroad, or from public or private research centers.
L'archive ouverte pluridisciplinaire HAL, est destinée au dépôt et à la diffusion de documents scientifiques de niveau recherche, publiés ou non, émanant des établissements d'enseignement et de recherche français ou étrangers, des laboratoires publics ou privés. 


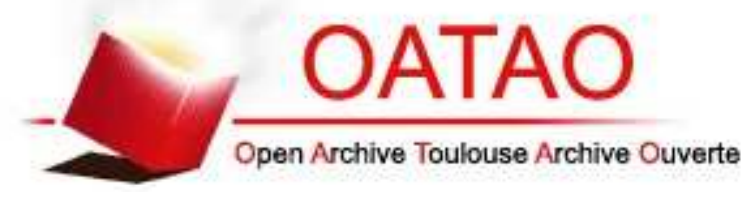

\section{Open Archive TOULOUSE Archive Ouverte (OATAO)}

OATAO is an open access repository that collects the work of Toulouse researchers and makes it freely available over the web where possible.

This is an author-deposited version published in : http://oatao.univ-toulouse.fr/ Eprints ID : 12890

The contribution was presented at ECAL 2013 :

http://www.dmi.unict.it/ecal2013/

To cite this version : Gürcan, Önder An Emergent Model for Mimicking Human Neuronal Pathways in Silico. (2013) In: 12th European Conference on Artificial Life (ECAL 2013), 2 September 2013 - 6 September 2013 (Taormina, Italy).

Any correspondance concerning this service should be sent to the repository administrator: staff-oatao@listes-diff.inp-toulouse.fr 


\title{
An Emergent Model for Mimicking Human Neuronal Pathways in Silico
}

\author{
Önder Gürcan ${ }^{1,2}$ \\ ${ }^{1}$ Ege University, Computer Engineering Department, Universite cad. 35100 Bornova, Izmir, Turkey \\ ${ }^{2}$ Paul Sabatier University, Institut de Recherche en Informatique de Toulouse, 118 route de Narbonne, 31062 Toulouse, France \\ onder.gurcan@\{ege.edu.tr,irit.fr\}
}

In this study, our aim is to mimick human neuronal pathways without assuming the transition from microscopic to macroscopic scales depend upon mathematical arguments. Human neuronal pathways are natural complex systems in which large sets of neurons interact locally and give bottomup rise to collective macroscopic behaviors. In this sense, correct knowledge of the synaptic effective connections between neurons is a key prerequisite for relating them to the operation of their central nervous system (CNS). However, estimating these effective connections between neurons in the human CNS poses a great challenge since direct recordings are impossible. Consequently, the network between human neurons is often expressed as a black box and the properties of connections between neurons are estimated using indirect methods (Türker and Powers, 2005). In indirect methods a particular receptor system is stimulated and the responses of neurons that are affected by the stimulus recorded to estimate the properties of the circuit. However, these neuronal circuits in human subjects are only estimations and their existence cannot be directly proven. Furthermore, there is no satisfactory theory on how these unknown parts of the CNS operate.

We propose a computational emergent model $^{1}$ that integrates the knowledge from neuroscience and artificial selforganization to derive from it the fundamental principles that govern CNS function and its simulation, and ultimately, to reconstruct the human CNS pathways in silico. The term artificial self-organization refers to a process enabling a software system to dynamically alter its internal organization (structure and functionality) during its execution time without any explicit external directing mechanism (Serugendo et al., 2011). Our emergent model uses temporal data collected from human subjects as an emergent macro-level description of the underlying neuronal pathway. Dynamic activity and spiking are modeled at the individual neuron scale. Consequently, the local information in the model is the knowledge about the behavior of individual neurons, such as generation of spikes and transmission of these spikes 2012). to their postsynaptic neurons. The effect of a spike on a target neuron is defined as a temporal membrane potential change in response to the influence of a source neuron that connects to it. That influence is not instantaneous, and is delayed by the physical distance between neurons. However, the interactions of neurons that result in macro-level emergent behaviors are unknown and obviously neurons alone are not able to deal with this information. Thus, we defined adaptive mechanisms for individual neurons based on biological knowledge. Moreover, to be able to specify purely local information about the reference macro-level pattern, we used the peristimulus frequency (PSF) analysis method (Türker and Powers, 2005).

We model the neuronal network as a dynamic directed graph $\mathcal{G}(t)=(\mathcal{N}(t), \mathcal{S}(t))$ where $\mathcal{N}(t)$ denotes the time varying cooperative neuron agent (vertex) set and $\mathcal{S}(t)$ denotes the time varying synapse (edge) set. The set of excitatory (resp. inhibitory) neuron agents at time $t$ is denoted by $\mathcal{N}^{+}(t)$ (resp. $\mathcal{N}^{-}(t)$ ) where $\mathcal{N}(t)=\mathcal{N}^{+}(t) \cup \mathcal{N}^{-}(t)$. A synapse $\{n, m\}$ delivers spikes from $n$ to $m$ with a delay of $d_{n m}$ and with a synaptic strength of $\eta$. We denote the set of postsynaptic neighbors of a neuron agent $n \in \mathcal{N}$ at time $t$ as $\operatorname{Post}_{n}(t)=\{k \in \mathcal{N}(t) \mid\{n, k\} \in \mathcal{S}(t)\}$ and the set of temporally closest presynaptic neighbors as $\operatorname{Temp}_{n}(t)$. The nominal behavior of neuron agents is spike firing. The adaptive behaviors of neuron agents are subjected to the non-cooperative situations of the agents which are propagated by the feedbacks (Algoritm 1). We denote the set of feedbacks as $\mathcal{F}$ and we model sending a feedback $f_{a} \in \mathcal{F}$ using an action of the form $\operatorname{send}\left(f_{a}, \mathcal{R}\right)$ where $a$ is the source of $f$ and $\mathcal{R} \subset \mathcal{N} \backslash\{a\}$ is the set of receiver agents. The tuning behavior of neuron agents is modelled using an action of the form tune $(\{n, m\}, f)$ for $n, m \in \mathcal{N}(t)$ and $f \in \mathcal{F}$, which correspond to the adjustment of $\{n, m\} . \eta$ by $f$ at time $t$. The reorganization behaviors of neuron agents are modeled using actions of the form $\operatorname{add}(\{n, m\})$ and remove $(\{n, m\})$ for $n, m \in \mathcal{N}(t)$, which correspond to the formation and suppression (respectively) of $\{n, m\}$ at time $t$. The evolution behaviors are modelled using actions of the form create $(n, m)$, createlnverse $(n, m)$ and remove $(n)$ 
for $n, m \in \mathcal{N}(t)$. create $(n, m)$ corresponds to the creation of a neuron agent between $n$ and $m$ by $n$ having the same type of $n$. createlnverse $(n, m)$ corresponds to the creation of a neuron agent between $n$ and $m$ by $n$ having the opposite type of $n$. remove $(n)$ corresponds to the suppression of the neuron agent $n$ by itself.

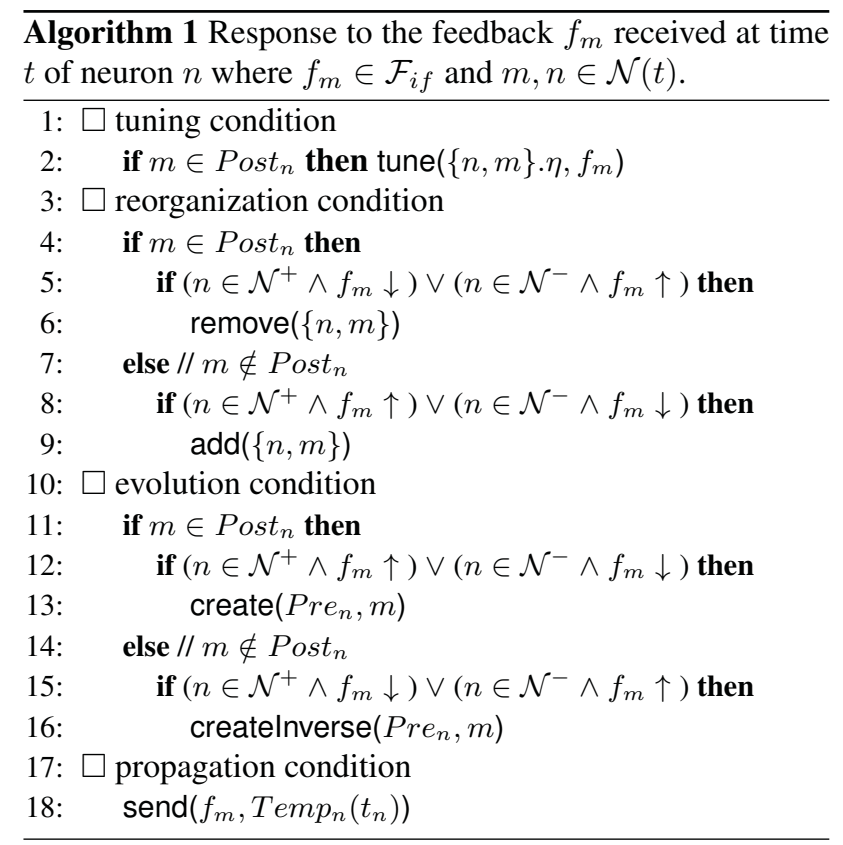

We applied the model to the reflex responses of single motor units obtained from conscious human subjects. The exact information we used about the underlying pathways is that sensory neurons make monosynaptic connections with the alpha motoneuron. In this sense, we considered this path as the shortest path in the underlying network and defined its duration as $l$. Therefore, we initialized the simulations as $\mathcal{N}(0)=\{s, m\}, \mathcal{S}(0)=\{\{s, m\},\{m, \varnothing\}\}$ and $d_{s m}=$ $d_{m \varnothing}=l / 2$ where $s, m \in \mathcal{N}^{+}$and $l$ is the latency of the estimated beginning of the pathway.

The results show that (e.g., Figure 1), the emergent neuronal network model learns to generate what is observed in human subjects in cellular resolution. What makes the model promising is the fact that, to the best of our knowledge, it is the first realistic model to self-wire an artificial neuronal network by efficiently combining neuroscience with artificial self-organization. Although there is no evidence yet of the model's connectivity mapping onto the human connectivity, we anticipate this model will help neuroscientists to learn much more about human neuronal networks, and could also be used for predicting hypotheses to lead future experiments.

\section{References}

Gürcan, Ö., Bernon, C., Türker, K. S., Mano, J.-P., Glize, P., and Dikenelli, O. (2012). Simulating human single motor units using self-organizing agents. In Self-Adaptive and SelfOrganizing Systems (SASO), 2012 IEEE Sixth International Conference on, pages $11-20$.

Gürcan, Ö., Türker, K. S., Mano, J.-P., Bernon, C., Glize, P., and Dikenelli, O. (2013). Mimicking human neuronal pathways in silico: an emergent model on the effective connectivity. Journal of Computational Neuroscience (accepted).

Serugendo, G., Gleizes, M.-P., and Karageorgos, A., editors (2011). Natural Computing Series. Springer.

Türker, K. S. and Powers, R. K. (2005). Black box revisited: a technique for estimating postsynaptic potentials in neurons. Trends in neurosci., 28(7):379-386.
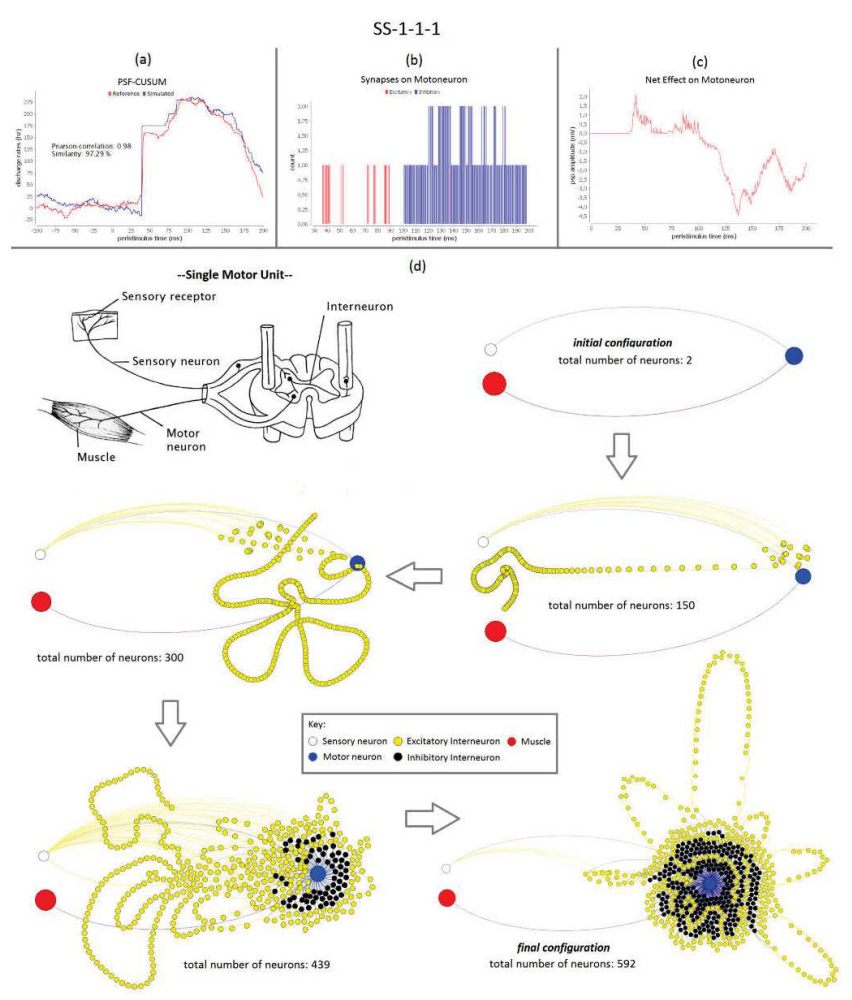

Figure 1: The results that came out of a simulation run at the end of its effort to learn the global pattern obtained from the human reflex experiment SS-1-1-1 (Gürcan et al., 2013). (a) PSF-CUSUM diagrams of the reference data (red line) and its simulated replication (blue line). Pearson-correlation of these lines is 0.98 and thus their similarity is $97.29 \%$. (b) The temporal distribution of created excitatory (red) and inhibitory (blue) synapses on the motoneuron. (c) The net PSP on motoneuron caused by its presynaptic connections given in (b). (d) The cinematic representation of the evolution of the neural network from the initial configuration towards the final configuration together with the number and the sign of neurons. Note also that, in the final configuration, the extent of the pathways that represent the long latency reflex responses are emerging as neuronal loops. 